CLINICAL STUDY

\title{
Increased carotid intima-media thickness in pre-pubertal children with constitutional leanness and severe obesity: the speculative role of insulin sensitivity, oxidant status, and chronic inflammation
}

\author{
C Giannini ${ }^{1}$, T de Giorgis ${ }^{1}$, A Scarinci ${ }^{2}$, I Cataldo ${ }^{3}$, M L Marcovecchio $^{1}$, F Chiarelli $^{1}$ and A Mohn ${ }^{1}$ \\ Departments of ${ }^{1}$ Pediatrics, ${ }^{2}$ Cardiology and ${ }^{3}$ Clinical Pathology, University of Chieti, Via dei Vestini 5, I- 66100 Chieti, Italy \\ (Correspondence should be addressed to A Mohn; Email: amohn@unich.it)
}

\begin{abstract}
Design: In order to characterize whether different degrees of adipose tissue storage may be associated with markers of early atherosclerosis, we evaluated oxidant-antioxidant status and inflammatory markers and determined carotid intima-media thickness (cIMT) in healthy constitutional lean and obese pre-pubertal children.

Methods: Eighty healthy pre-pubertal lean and obese children were recruited and compared with 40 age, gender, and pubertal stage-matched normal controls. Anthropometric measurements, oxidant (urinary isoprostanes (PGF-2 $\alpha$ ), lag phase, and malondialdehyde (MDA)) and antioxidant status (vitamin E), inflammatory markers (high sensitive C-reactive protein (hs-CRP)), and insulin sensitivity (fasting glucose-insulin ratio, homeostasis model assessment of insulin resistance (HOMA-IR)) were investigated. Furthermore, cIMT was measured by high-resolution ultrasound.

Results: hs-CRP was not different between lean and control subjects $(P=0.45)$, while higher values were found in obese compared with lean and control children $(P<0.001$ and $P<0.001$ respectively). PGF- $2 \alpha$ and MDA were higher while lag phase shorter in lean and obese subjects compared with controls (lean $P<0.001 ; P<0.001 ; P<0.001$ and obese $P<0.001 ; P<0.001 ; P<0.001$ respectively), while no differences were documented between lean and obese subjects $(P=0.78$, $P=0.019$, and $P=0.53$ respectively). Compared with controls, cIMT was increased in lean and in obese subjects $(P=0.001 ; P=0.004)$, while no differences were documented between obese and lean subjects $(P=0.1)$. In a multiple stepwise linear regression analysis, cIMT was related with PGF- $2 \alpha$ $(\beta=0.641, P<0.001)$ and HOMA-IR $(\beta=0.307 ; P<0.001)$.

Conclusions: Pre-pubertal lean and obese children present increased oxidative stress and impaired inflammation and insulin sensitivity, which in turn seem to result in similar impaired endothelial dysfunction and early signs of atherosclerosis, already in childhood.
\end{abstract}

European Journal of Endocrinology 161 73-80

\section{Introduction}

During the past decades, several studies have established an important role of adipose tissue on the development of cardiovascular diseases (1-4). A number of epidemiological studies have demonstrated an increased morbidity and mortality for cardiovascular disease that appears to be associated with extremely obese as well as lean subjects (1-4). Subjects with body mass index (BMI) of $22.2 \mathrm{~kg} / \mathrm{m}^{2}$ have the lowest morbidity compared with those who have both higher and lower values suggesting an important role of adipose tissue on cardiovascular events (2).

Adipose tissue has been considered a proper endocrine tissue able to synthesize several molecules, which induce impaired metabolic status or determine important antiatherogenic, anti-inflammatory, and antidiabetic effects (5). The exposure during childhood to these metabolic alterations may induce changes in the arteries contributing to the development of atherosclerosis in adulthood (6). High-resolution B-mode ultrasound measurements of the carotid intima-media thickness (cIMT) are a feasible, direct, and non-invasive method able to evaluate and detect preclinical atherosclerosis. Studies in childhood showed significant increased cIMT in obese children (7-9), while, to the best of our knowledge, no studies have been performed in lean children.

There are substantial evidences that obesity in childhood lays the metabolic groundwork for adult cardiovascular disease $(6,10-12)$ where the obese related increase in adipose tissue appears to be the first 
step in promoting the initial abnormalities in glucose metabolism and in the development of insulin resistance (IR). The induction of IR appears to sustain an increase in different inflammatory markers (13) associated with impaired oxidative stress, which in turn seem to play the pivotal role in the early stages of atherogenesis, including impairment of endothelial functions and the formation of fatty streaks and plaques $(9,14,15)$. Vice versa in lean adult subjects, similar pro-atherosclerotic alterations consisting of increased IR and impaired oxidant/antioxidant status have been demonstrated (16-20). As this was also associated with impaired endothelial function (20), it might be argued that the lack of adipose tissue represents a trigger for the initiation of an atherosclerotic process by inducing peripheral IR, which seems to be secondary to different mechanisms (16-19) such as aberrant storage of triglycerides (TGs) in other organs (i.e. liver and muscles); increased free fatty acid flux leading to altered glucose uptake by the skeletal muscle and increased hepatic glucose output; as well as impaired adipocytokines production (especially leptin and adiponectin).

The present study aimed to verify whether constitutional lean pre-pubertal children present impaired oxidant/antioxidant status, IR, and chronic inflammation resulting in differences in cIMT compared with obese and control subjects.

\section{Materials and methods}

\section{Study population}

Forty lean pre-pubertal children who had been referred to the Endocrine Clinic of the Department of Paediatrics, University of Chieti, Italy, were recruited according to BMI values. Only subjects with BMI lower than the 2 SDS for the mean age and gender and with constitutional leanness were eligible, while those with secondary leanness were excluded (defined by thyroid dysfunction, celiac disease, chronic diarrhoea, GH/ insulin-like growth factor-1 axis alteration, and anorexia). Furthermore, 40 obese pre-pubertal children were recruited according to BMI values. All obese subjects were affected by severe essential obesity (BMI higher than 2 SDS, for the mean age and gender) (21). Eligible obese and lean subjects were appropriate for gestational age at birth and did not have other chronic diseases (diabetes mellitus, celiac disease, thyroid dysfunction, endocrine disorders, hereditary disease, or systemic or bowel inflammatory disease, and malabsorption) as well as not taking any medication. Subjects with severe dyslipidemia (TGs $>600 \mathrm{mg} / \mathrm{dl}$ or cholesterol $>300 \mathrm{mg} / \mathrm{dl}$ ) and ingestion of vitamin E during the previous 4 months were excluded. As a control group, we recruited 40 healthy pre-pubertal children comparable for age, gender, and pubertal stage with a BMI between -2 and +2 SDS, who were admitted to the
Department of Paediatrics of the University of Chieti for minor diseases. Blood and urinary sample, anthropometric and instrumental measurements were taken only after complete recovery of those diseases.

A complete physical examination was performed, including anthropometric parameters (height and weight), and staging of puberty on the basis of the breast development in girls and genital development in boys according to the Tanner's criteria. We analyzed also fatness indices (BMI, BMI-SDS, waist and hip circumference (WC and $\mathrm{HC}$ ), waist-to-hip ratio (WHR), and skin-fold thicknesses) and basal blood pressure.

Venous fasting blood samples to evaluate inflammatory markers (high sensitive C-reactive protein (hs-CRP)), lipid profile (total cholesterol, low density lipoprotein (LDL)-cholesterol, and TGs) and plasma glucose and insulin. Homeostasis model assessment of IR (HOMA-IR) and fasting glucose-insulin ratio (G/I) were calculated as markers of insulin sensitivity.

Urinary isoprostanes (PGF-2 $\alpha$ ) as well as lag phase and malondialdehyde (MDA) were analyzed as markers of oxidative stress, while plasma vitamin E content was evaluated in order to characterize the antioxidant status. Furthermore, both right and left cIMT was measured by a high B-mode ultrasonography.

This study was approved by Ethical Committee of University of Chieti. Written informed consent was obtained from all parents and oral consent from all children.

\section{Anthropometric measurements}

Body weight was determined to the nearest $0.1 \mathrm{~kg}$, and height was measured in triplicate with a Harpenden stadiometer to the nearest $0.1 \mathrm{~cm}$.

As fatness indices, we used BMI (the weight in kilograms divided by the square of the height in meters), and the BMI-SDS for age and gender was calculated (22). The distribution of fat mass was valued with WHR (WC was measured at its smallest point between iliac crest and rib cage while HC at its largest width over the greater trochanters). Additionally, we used fat mass $\%$, estimated from four skin-fold thickness (made over the triceps and biceps, at subscapular and abdominal, of the left side of the body) with a Holtain plicometer according to Brook's equation, skin-fold measurements being more feasible in small children. Skin-fold thicknesses were measured as previously described (23).

\section{Laboratory procedures}

Biochemical analysis Fasting plasma glucose level was determined by using the glucose oxidase method, and plasma insulin was measured with two-site immunoenzymometric assay (AIA-PACK IRI; Tosoh, Tokyo, Japan). For insulin, the limit of detection was $0.5 \mu \mathrm{U} / \mathrm{ml}$ with intra- and interassay coefficients of 
variation $<7 \%$ for quality control. We used the following indices for determination of IR: baseline G/I; HOMA-IR calculated with the formula: fasting insulin $(\mathrm{mU} / \mathrm{l}) \times$ fasting glucose $(\mathrm{mmol} / \mathrm{l}) / 22.5$.

Lipid analysis Fasting serum total cholesterol and TGs concentrations were determined by colorimetric enzymatic method. LDL-cholesterol was measured by a commercially available enzymatic reagent (CHOD-PAP, MPR1, Boeringer Mannheim).

\section{LDL isolation and oxidation}

Fasting venous blood was taken from subjects and placed in tubes containing EDTA (2.7 $\mathrm{mmol} / \mathrm{l})$, and plasma was immediately separated by centrifugation. The LDL fraction was isolated from freshly drawn plasma as previously described (24). To protect LDL against oxidative modification during isolation, EDTA ( $2.7 \mathrm{mmol} / \mathrm{l})$ was added to density solutions. Oxidation of LDL (fresh preparations at a concentration of $0.2 \mathrm{mg}$ $\mathrm{LDL}$ cholesterol $/ \mathrm{ml}$ ) was triggered by the addition of $5 \mu \mathrm{mol} / \mathrm{I} \mathrm{CuSO}_{4}$ in PBS, pH 7.4, at $37^{\circ} \mathrm{C}$ and continuously monitored spectrophotometrically at $234 \mathrm{~nm}$ to evaluate the formation of conjugated dienes. Oxidation of LDL was calculated as the measurement of the duration of the phase before the maximum oxidation. The oxidation curve is characterized by the lag phase, the propagation phase, and the decomposition phase (25). In particular, lag phase is the time required by the reaction to gain the maximum velocity of $V_{\max }$ during propagation phase (25). Lag phase and propagation rate were calculated as previously described (25).

\section{Peroxidation of $L D L$}

LDL peroxidation was evaluated spectrophotometrically by the measurement of the MDA using the thiobarbituric acid-reacting substance assay as previously described (26). Results were expressed as MDA (nmol)/LDL protein (mg) (26).

\section{Urinary isoprostanes}

Multiple aliquot of early morning urine samples was added with the antioxidant 4-hydroxy-tempo (Sigma Chemical Co.) and stored at $-80^{\circ} \mathrm{C}$. PGF- $2 \alpha$ were evaluated in triplicate by immunoenzymatic methods (ELISA, Oxford Biomedical Research, Enzyme Immunoassay for urinary isoprostane) (27).

\section{Vitamin E determination}

Plasma vitamin E, expressed in $\mu \mathrm{mol} / \mathrm{l}$, was measured in duplicate with HPLC using a Kontrol System 450 (Milan, Italy) equipped with an u.v.-visible spectrophotometer (Kontrol Detector 430) at different wavelengths. Procedures were as previously reported (28).

\section{High sensitive C-reactive protein}

Multiple plasma sample aliquots were collected and stored at $-80{ }^{\circ} \mathrm{C}$ until analysis. hs-CRP was measured by latex-enhanced nephelometry (N High Sensitivity CRP assay) on a BN nephelometer (Dade Behring Inc, Marburg, Germany). The lower limit of detection of this assay was $0.1 \mathrm{mg} / \mathrm{l}$ (29).

\section{Instrumental procedures}

Blood pressure The blood pressure was measured in children by one investigator using a validation protocol. Systolic blood pressure (SBP) and diastolic blood pressure (DBP) were measured twice according to the previously reported guideline. Hypertension was defined as blood pressure values above the 95th percentile for height, age, and gender (30).

Carotid ultrasonography High-resolution B-mode ultrasonography of the right and left carotid artery was performed with a linear $14 \mathrm{mHz}$ transducer (Philips Sonos). The subjects were examined in the supine position with the head turned slightly to the left and right. The common, internal, and external carotid arteries were identified by combined B-mode and colordoppler ultrasound examinations. A careful search was performed to obtain an optimal visualization of the vessel wall demonstrating the typical double lines representing the intima-media layer. Intima-media thickness was defined as the distance between the leading edge interface of the far wall and the leading edge of the median adventitia interface of the far wall with an automated edge-tracking method, as previously described (31). Three determinations of the right and the left carotid artery were performed and these three determinations were averaged; furthermore, the right and the left carotid artery measurements were averaged and used for analysis (mean cIMT). During the ultrasound, scanning images were digitally frozen and printed. The measurements were performed by the same operator who was blinded to the study design. For quality control assessment, $10 \%$ of the subjects were re-examined and these repeated measurements gave a mean difference of $0.01 \pm 0.01 \mathrm{~mm}$ and a coefficient of variation of $1 \%$.

\section{Statistical analysis}

All values were expressed as mean and s.D. Differences in gender variable were analyzed by $\chi^{2}$ test. Differences between the three groups were tested by one-way ANOVA with Bonferroni's test for post hoc analysis. The statistical significance level was $P<0.05$. Furthermore, adjustment for potential confounding factors (age, gender, BMI-SDS, blood pressure, and LDL-cholesterol) was performed using analysis of covariance. 
Within lean subjects, to investigate the independent effect of IR and inflammatory and oxidative stress status on the increasing cIMT, a correlation between these parameters was detected by multiple stepwise linear regression analysis (PGF-2 $\alpha$, hs-CRP, HOMA-IR, age, gender, BMI-SDS, SBP, and LDL-cholesterol). The statistical significance level was $P<0.05$.

All calculations were made with the computer program SPSS (Statistical Package for the Social Science Inc, Chicago, United States), version 14.0 software for Windows.

\section{Results}

Forty pre-pubertal constitutional lean children and 40 obese children were recruited and compared with 40 healthy subjects similar for age, gender, and pubertal stage. The mean clinical characteristic and levels of biochemical parameters are summarized in Table 1.

\section{Anthropometric measurements}

Significant differences were documented among the three groups according to BMI, BMI-SDS, skin-fold thickness (biceps, triceps, subscapular, and abdominal), fat mass, fat mass \%, WC, HC, and WHR (Table 1). In detail, compared with controls and obese subjects, lean children had significantly lower BMI $(P<0.001$ and $P<0.001$ respectively), BMI-SDS values $(P=0.005$ and $P=0.001$ respectively), skin-fold thickness, and fat mass $\%$ (versus controls: biceps $P=0.011$; triceps $P<0.001$; abdominal $P<0.001$; subscapular $P<0.001$; fat mass \% $P<0.001$ respectively; and versus obese biceps

Table 1 Baseline clinical characteristics and levels of biochemical parameters of lean, obese, and normal control pre-pubertal children.

\begin{tabular}{|c|c|c|c|c|}
\hline & Lean & Normal controls & Obese & $P^{a}$ \\
\hline \multicolumn{5}{|l|}{ Auxology } \\
\hline Number & 40 & 40 & 40 & \\
\hline Age (years) & $8.7 \pm 1.5$ & $8.1 \pm 1.1$ & $8.5 \pm 1.7$ & NS \\
\hline Gender (M/F) & $22 \mathrm{M} / 18 \mathrm{~F}$ & $24 \mathrm{M} / 16 \mathrm{~F}$ & $19 \mathrm{M} / 21 \mathrm{~F}$ & $0.1^{\mathrm{b}}$ \\
\hline Weight (kg) & $22.7 \pm 6.1$ & $25.7 \pm 7.4$ & $45.6 \pm 14.4$ & $<0.001^{*, t, \neq}$ \\
\hline $\mathrm{BMI}\left(\mathrm{kg} / \mathrm{m}^{2}\right)$ & $13.5 \pm 0.9$ & $16.5 \pm 0.8$ & $25.2 \pm 4.7$ & $<0.001^{*,+, \ddagger}$ \\
\hline BMI-SDS & $-2.17 \pm 0.08$ & $0.54 \pm 0.9$ & $5.9 \pm 2.0$ & $<0.001^{*, t, \neq}$ \\
\hline Fat mass $\%$ & $13.6 \pm 4.1$ & $20.69 \pm 7.7$ & $41.5 \pm 7.9$ & $<0.001^{*, t, \neq}$ \\
\hline Skin-fold thicknesses bicipital & $8.0 \pm 2.4$ & $9.2 \pm 2.8$ & $19.68 \pm 6.9$ & $<0.001^{*,+, \neq}$ \\
\hline Skin-fold thicknesses scapular & $4.38 \pm 1.5$ & $6.67 \pm 1.9$ & $21.75 \pm 9.3$ & $<0.001^{*, t, \ddagger}$ \\
\hline Skin-fold thicknesses triceps & $5.24 \pm 1.9$ & $9.83 \pm 2.9$ & $25.34 \pm 6.1$ & $<0.001^{*, t, \neq}$ \\
\hline Skin-fold thicknesses abdominal & $4.6 \pm 1.1$ & $7.04 \pm 2.9$ & $20.8 \pm 8.3$ & $<0.001^{*, t, \neq}$ \\
\hline WC $(\mathrm{cm})$ & $50.0 \pm 5.8$ & $54.8 \pm 5.7$ & $77.2 \pm 13.4$ & $<0.001^{*, t, \neq}$ \\
\hline $\mathrm{HC}(\mathrm{cm})$ & $59.8 \pm 5.8$ & $63.4 \pm 8.5$ & $83.1 \pm 10.8$ & $<0.001^{\dagger, \ddagger}$ \\
\hline WHR & $0.84 \pm 0.04$ & $0.86 \pm 0.04$ & $0.92 \pm 0.05$ & $<0.001^{\dagger, \ddagger}$ \\
\hline $\mathrm{SBP}(\mathrm{mmHg})$ & $102 \pm 11$ & $97 \pm 9$ & $109 \pm 12$ & $<0.001^{\ddagger}$ \\
\hline $\mathrm{DBP}(\mathrm{mmHg})$ & $64 \pm 13$ & $62 \pm 7$ & $67 \pm 6$ & $<0.001^{\ddagger}$ \\
\hline \multicolumn{5}{|l|}{ cIMT } \\
\hline Right clMT (mm) & $0.37 \pm 0.01$ & $0.31 \pm 0.04$ & $0.40 \pm 0.06$ & $<0.001^{*, \neq}$ \\
\hline Left cIMT (mm) & $0.38 \pm 0.01$ & $0.31 \pm 0.06$ & $0.40 \pm 0.06$ & $<0.001^{* \neq}$ \\
\hline Mean cIMT (mm) & $0.37 \pm 0.01$ & $0.31 \pm 0.06$ & $0.40 \pm 0.06$ & $<0.001^{*, \neq}$ \\
\hline \multicolumn{5}{|l|}{ Lipid profile } \\
\hline Total cholesterol (mg/dl) & $169 \pm 25$ & $159 \pm 23$ & $169 \pm 19$ & NS \\
\hline LDL-cholesterol (mg/dl) & $98 \pm 20$ & $96 \pm 10$ & $102 \pm 11$ & NS \\
\hline $\mathrm{TG}(\mathrm{mg} / \mathrm{dl})$ & $83 \pm 40$ & $70 \pm 27$ & $101 \pm 54$ & NS \\
\hline \multicolumn{5}{|l|}{ IR } \\
\hline Fasting insulin $(\mu \mathrm{U} / \mathrm{ml})$ & $8.9 \pm 5.3$ & $5.3 \pm 2.0$ & $10.9 \pm 6.6$ & $0.001^{*, \neq}$ \\
\hline Fasting glycemia (mg/dl) & $80.6 \pm 7.9$ & $78.3 \pm 8.3$ & $86.5 \pm 8.1$ & $0.002^{\dagger, \ddagger}$ \\
\hline C-peptide (mg/dl) & $0.86 \pm 0.36$ & $0.86 \pm 0.68$ & $1.42 \pm 0.72$ & $<0.001^{*, \mp}$ \\
\hline HOMA-IR & $1.82 \pm 1.1$ & $1.05 \pm 0.4$ & $2.36 \pm 1.4$ & $<0.001^{*, \neq}$ \\
\hline $\mathrm{G} / \mathrm{I}$ & $13.3 \pm 8.3$ & $17.04 \pm 7.9$ & $11.8 \pm 7.8$ & $0.003^{\star \neq}$ \\
\hline \multicolumn{5}{|l|}{ Oxidant status } \\
\hline hs-CRP (mg/l) & $0.24 \pm 0.3$ & $0.42 \pm 0.2$ & $2.6 \pm 1.3$ & $P<0.001^{\dagger, \ddagger}$ \\
\hline PGF-2 $\alpha$ (ng/ml) & $7.0 \pm 2.6$ & $2.5 \pm 2.0$ & $6.9 \pm 3.2$ & $P<0.001^{*, \neq}$ \\
\hline Lag phase (min) & $33.4 \pm 7.5$ & $47.9 \pm 6.9$ & $27.5 \pm 16.6$ & $P<0.001^{*, \neq}$ \\
\hline MDA (nmol/mg) & $0.69 \pm 0.15$ & $0.54 \pm 0.18$ & $0.79 \pm 0.22$ & $P<0.001^{*, \ddagger}$ \\
\hline Vit. $E(\mu \mathrm{mol} / \mathrm{l})$ & $36.2 \pm 10.5$ & $36.3 \pm 7.3$ & $35.9 \pm 8.7$ & NS \\
\hline
\end{tabular}

*Significant differences by post hoc analysis $(P<0.05)$ (lean versus controls). ${ }^{\dagger}$ Significant differences by post hoc analysis $(P<0.05$; lean versus obese). ${ }^{\ddagger}$ Significant differences by post hoc analysis $(P<0.05)$ (obese versus controls). NS, no significant difference $(P>0.05)$. Values are mean \pm S.D. SDS, S.D. score; BMI, body mass index; WC, waist circumference; HC, hip circumference; WHR, waist-to-hip ratio; SBP, systolic blood pressure; DBP, diastolic blood pressure; cIMT, carotid intima-media thickness; TG, triglyceride; HOMA-IR, homeostasis model assessment of insulin resistance; G/I, fasting glucose-insulin ratio; hs-CRP, high sensitivity c-reactive protein; PGF- $2 \alpha$, urinary isoprostanes; MDA, malondialdehyde; Vit $\mathrm{E}$, plasma vitamin $\mathrm{E}$.

aOne-way ANOVA.

${ }^{\mathrm{b}} \chi^{2}$ test. 
$P<0.011$; triceps $P<0.001$; abdominal $P<0.001$; subscapular $P<0.001$; fat mass $\% P<0.001$ respectively), while these parameters were significantly higher in obese than controls $(P=0.003$ and $P=0.005$, biceps, triceps, abdominal, subscapular, and fat mass $\%=$ $P<0.001$ respectively). In addition, $\mathrm{WC}, \mathrm{HC}$, and WHR were significantly lower in lean children compared with obese $(P<0.001, P<0.001$, and $P<0.001$ respectively $)$ and higher in obese compared with controls $(P<0.001$, $P<0.001$, and $P<0.001$ respectively). Compared with controls, WC was significantly lower in lean subjects $(P=0.001)$, while HC and WHR were similar $(P=0.13$ and $P=0.07$ respectively).

SBP and DBP results were statistically different among the three groups (Table 1). In detail, no significant differences were documented for SBP and DBP in lean children compared with controls $(P=0.03$ and $P=0.47$ respectively) and obese $(P=0.08$ and $P=0.37$ respectively), while obese children showed higher SBP and DBP compared with controls $(P<0.001$ and $P<0.001)$.

\section{Lipid profile}

No difference was found in terms of total cholesterol, LDL-cholesterol, and TG, among the three groups (Table 1).

\section{Oxidant-antioxidant status}

Significant differences were documented among the three groups according to hs-CRP, PGF- $2 \alpha$, lag phase, and MDA (Table 1), while vitamin E levels were similar. In detail, hs-CRP was higher in obese children compared with lean and control children $(P<0.001$ and $P<0.001$ respectively), while no differences were found between lean children compared with controls $(P=0.45)$. Furthermore, PGF- $2 \alpha$ and MDA were higher while lag phase was shorter in lean and obese subjects compared with controls (lean $P<0.001 ; P<0.001$; $P<0.001$ and obese $P<0.001 ; P<0.001 ; P<0.001$ respectively), whereas no differences were documented between lean and obese subjects $(P=0.78, P=0.019$, and $P=0.53$ respectively).

\section{Insulin-resistance indices}

Fasting insulin, fasting glycemia, HOMA-IR, and G/I results were significantly different among the three groups (Table 1). Lean and obese subjects had higher fasting insulin, fasting glycemia, HOMA-IR (lean $P=0.002 ; P=0.013 ; P=0.011$ and obese $P<0.001$; $P<0.001, P<0.001$ respectively), and lower $G /$ I values compared with controls $(P=0.011$, and $P=0.001$ respectively); while no differences were documented between lean and obese $(P=0.19 ; P=0.019 ; P=0.08$; $P=0.35$ respectively).

\section{cIMT}

Significant differences were documented in left, right, and mean cIMT among the three groups (Table 1). In details compared with controls, the mean value of the left and the right carotid artery was higher in lean and obese children (mean cIMT $P<0.001$ and $P<0.001$ respectively), while no significant differences were found between lean and obese (mean cIMT $P=0.1$ ). Across the whole population, the mean value of the left and the right carotid artery showed a characteristic J-curve distribution with higher values in the lower and higher part of the BMI (Fig. 1). After adjustment for gender, age, BMI-SDS, SBP, and LDL-cholesterol, the difference in mean cIMT in both lean and obese children remains statistically significant compared with the control group $(P<0.05$ for trend).

\section{Multiple regression analysis}

In order to investigate the independent effect of IR and oxidative stress status on cIMT, a multiple stepwise regression analysis was performed. The mean cIMT was significantly related with PGF-2 $\alpha \quad(\beta=0.639$, $P<0.0001)$ and HOMA-IR $(\beta=0.397 ; P<0.001)$, whereas there were no correlations between cIMT and age, gender, BMI-SDS, SBP, LDL-cholesterol, and hs-CRP.

\section{Discussion}

This is the first study of a comparable negative effect of both lower and higher adipose tissue storage on carotid arterial wall in constitutional lean and severe obese prepubertal children.

Functional and morphological alterations of the vascular wall are universally accepted as an early and

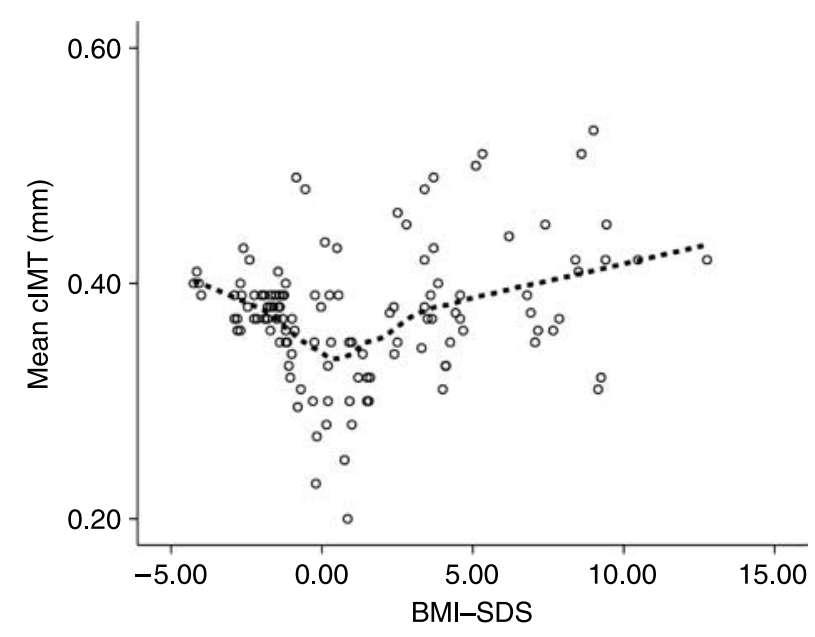

Figure 1 Distribution of the mean value of the left and the right carotid intima-media thickness (mean cIMT) across the lean, control, and obese pre-pubertal children. 
pivotal step in the development of atherosclerosis, and the detected increased cIMT could reflect these structural changes (32-34). Previous reports have indicated that cIMT is associated with coronary artery diseases and cardiovascular morbidity and mortality (32-34). Studies in childhood showed significantly increased cIMT in children with hypertension (35), type 1 diabetes (36), familial hypercholesterolemia (37), and severe obese children (9), while no data on increased cIMT in lean children are reported. Furthermore, in line with previous studies on a similar impaired degree of endothelial function in both obese and lean young adults (20), we have demonstrated for the first time a significant increase of cIMT in lean pre-pubertal children compared with controls. Moreover, although both lean and obese children showed significantly higher cIMT compared with controls, no differences were found between the two groups. The documented increase in cIMT in our population is important as it concerns only healthy pre-pubertal children helping to avoid any pubertal-related effects that could influence the precocious impairments of the arterial wall (38). Furthermore, we have excluded other factors such as hypertension, hypercholesterolemia, and smoking, which are well known to induce early alterations of the arterial wall. However, a systematic longitudinal follow-up of these pre-pubertal children is necessary in order to confirm unequivocally the role of the revealed differences of cIMT on cardiovascular disease in adulthood.

Adipose tissue accumulation in patients with generalized or regional obesity and markedly adipose tissue deficiency in patients with genetic or acquired lipodystrophy are both associated with IR and its complications (e.g. type 2 diabetes, hypertriglyceridemia, low levels of high-density lipoprotein cholesterol, and hepatic steatosis) (39-41). In this study, we have demonstrated an impaired insulin sensitivity in both obese and lean pre-pubertal children compared with controls, while no difference was detected between lean and obese subjects, underling a similar effect of increased and decreased adipose tissue reserve on changes in IR. Although relevant, the underlying mechanisms by which the lack of adipose tissue causes IR remain not completely elucidated and several hypotheses have been proposed $(16-19,39-41)$. First, patients with lipodystrophies are lacking sufficient adipocytes, and, therefore, the aberrant lipid flux results in a variant storage of fat in non-lipodystrophic tissues, such as the liver and skeletal muscles interfering with insulin responsiveness (at the liver and skeletal muscle) or with altered glucose-stimulated insulin secretion (in pancreatic $\beta$-cell) (17). Another hypothesis relates to a possible reduced glucose uptake by skeletal muscles and increased hepatic glucose output due to increased FFA flux in patients with partial lipodystrophies $(18,19)$. A third hypothesis pertains to the role of adipocytokines, the secretory products of adipose tissue, in causing IR
(18, 19). For example, patients with generalized lipodystrophies have deficiencies of leptin and adiponectin, which may be related to severe IR (19). However, as in this study no information on adipokines or ultrasound determination of aberrant storage TGs in other organs was evaluated, further longitudinal studies are needed in order to offer more information on the underlying relevant mechanisms.

Several studies have clearly demonstrated a tight association between adiposity-related increased IR, oxidative stress, and inflammation in adults as well as in children $(9,11,32)$. Impaired insulin sensitivity has continuously been considered to be an important promoting factor of atherosclerosis $(11,42)$ through chronic inflammation and impaired oxidative stress, two well-known mechanisms directly involved on the development of endothelial dysfunction in both obese adults and children $(9,14,29,43)$. Especially, PGF- $2 \alpha$ can induce vasoconstriction in many vascular beds, promote platelet aggregation, and support proliferation of vascular smooth muscle cells, and they have been considered an important proatherogenic factor on the arterial wall (44-47). We have demonstrated for the first time that lean pre-pubertal children present an impaired oxidant/antioxidant status compared with controls. In fact, lean children showed a significant increase in oxidative markers compared with age, gender, and pubertal stage-matched controls. However, although both lean and obese children showed significantly impaired oxidative status compared with controls, no differences were found between the two groups. In fact, in lean pre-pubertal children, oxidative markers (urinary PGF- $2 \alpha$, lag phase, MDA, and vitamin E) were similar to those detected in obese children demonstrating similar effects of extremely low or high adipose tissue storage. Impaired insulin sensitivity as well as oxidative stress/inflammatory status has been demonstrated to be directly related to early arterial changes in obese children and adults $(9,11,32,42)$. Similar to those observed in obese subjects, by inducing IR and impaired oxidative status, adipose tissue deficiency in lean children appears to influence changes in the vascular wall. In fact, by multiple stepwise linear regression analysis, we documented an independent effect of IR and oxidative stress status on the increasing cIMT within lean and obese subjects. In fact, cIMT was tightly associated with HOMA-IR, PGF- $2 \alpha$, and lag phase. According to these results, our data suggest that impaired insulin sensitivity and altered oxidant-antioxidant status, related to adipose tissue depletion, represent key elements for the development of early abnormalities in the arterial wall not only in obese subjects but also in lean pre-pubertal children. These findings represent the first report on the relationship between low adipose tissue reserve and cIMT. Our data appear to be supported by previous studies that reported a J-curve phenomenon between prevalence of disease, including cardiovascular complications, and BMI 
suggesting a direct role of adiposity on several diseases including cardiovascular events (1-4). Therefore, obesity as well as leanness appears to similarly induce impaired IR, oxidative stress, and chronic inflammation, which in turn induce endothelial dysfunction leading to cIMT, an early atherosclerotic change in the arterial wall that is universally accepted as a cardiovascular risk factor. However, as this study is a cross-sectional study, further data from a longitudinal study or intervention study are needed in order to confirm this observation.

In conclusion, pre-pubertal lean and obese children present increased oxidative stress and impaired inflammation and insulin sensitivity, which in turn seem to result in a similar impaired endothelial dysfunction and early sign of atherosclerosis. Therefore, impaired adipocyte stores determine an unbalanced endothelial regulation resulting in an increased risk of cardiovascular complications. A complete clarification of the adipose tissue-related endocrine effects represents an important action on the developing of new targets for therapeutic approach in order to prevent and restore the adiposity-related impaired status, early in childhood.

\section{Declaration of interest}

All authors declare that there is no conflict of interest that could be perceived as prejudicing the impartiality of the research reported.

\section{Funding}

This research did not receive any specific grant from any funding agency in the public, commercial, or not-for-profit sector.

\section{Acknowledgements}

We thank all nurses of the Department of Pediatrics, University of Chieti, and in particular Di Santo D, Di Domenica S, Finamore C, for their daily support in clinical research and care of children.

\section{References}

1 Adams KF, Schatzkin A, Harris TB, Kipnis V, Mouw T, BallardBarbash R, Hollenbeck A \& Leitzmann MF. Overweight, obesity, and mortality in a large prospective cohort of persons 50 to 71 years old. New England Journal of Medicine 2006355 763-778.

2 Tokunaga K, Matsuzawa Y, Kotani K, Keno Y, Kobatake T, Fujioka S \& Tarui S. Ideal body weight estimated from the body mass index with the lowest morbidity. International Journal of Obesity 199115 1-5.

3 Breeze E, Clarke R, Shipley MJ, Marmot MG \& Fletcher AE. Causespecific mortality in old age in relation to body mass index in middle age and in old age: follow-up of the Whitehall cohort of male civil servants. International Journal of Epidemiology 200635 169-178.

4 Pischon T, Boeing H, Hoffmann K, Bergmann M, Schulze MB, Overvad K, van der Schouw YT, Spencer E, Moons KG, Tjønneland A, Halkjaer J, Jensen MK, Stegger J, ClavelChapelon F, Boutron-Ruault MC, Chajes V, Linseisen J, Kaaks R, Trichopoulou A, Trichopoulos D, Bamia C, Sieri S, Palli D, Tumino R, Vineis P, Panico S, Peeters PH, May AM, Buenode-Mesquita HB, van Duijnhoven FJ, Hallmans G, Weinehall L, Manjer J, Hedblad B, Lund E, Agudo A, Arriola L, Barricarte A,
Navarro C, Martinez C, Quirós JR, Key T, Bingham S, Khaw KT, Boffetta P, Jenab M, Ferrari P \& Riboli E. General and abdominal adiposity and risk of death in Europe. New England Journal of Medicine 2008359 2105-2120.

5 Katagiri H, Yamada T \& Oka Y. Adiposity and cardiovascular disorders: disturbance of the regulatory system consisting of humoral and neuronal signals. Circulation Research 2007101 27-39.

6 Berenson GS, Srinivasan SR, Bao W, Newman WP III, Tracy RE \& Wattigney WA. Association between multiple cardiovascular risk factors and atherosclerosis in children and young adults. The Bogalusa Heart Study. New England Journal of Medicine 1998338 1650-1656.

7 Tounian P, Aggoun Y, Dubern B, Varille V, Guy-Grand B, Sidi D, Girardet JP \& Bonnet D. Presence of increased stiffness of the common carotid artery and endothelial dysfunction in severely obese children: a prospective study. Lancet 2001358 1400-1404.

8 Reinehr T, Kiess W, de Sousa G, Stoffel-Wagner B \& Wunsch R. Intima media thickness in childhood obesity: relations to inflammatory marker, glucose metabolism, and blood pressure. Metabolism 200655 113-118.

9 Giannini C, de Giorgis T, Scarinci A, Ciampani M, Marcovecchio ML, Chiarelli F \& Mohn A. Obese related effects of inflammatory markers and insulin resistance on increased carotid intima media thickness in pre-pubertal children. Atherosclerosis $2008197448-456$.

10 Hannon TS, Rao G \& Arslanian SA. Childhood obesity and type 2 diabetes mellitus. Pediatrics 2005116 473-480.

11 Steinberger J \& Daniels SR. Obesity, insulin resistance, diabetes, and cardiovascular risk in children: an American Heart Association scientific statement from the Atherosclerosis, Hypertension, and Obesity in the Young Committee (Council on Cardiovascular Disease in the Young) and the Diabetes Committee (Council on Nutrition, Physical Activity, and Metabolism). Circulation 2003107 1448-1453.

12 Freedman DS, Dietz WH, Tang R, Freedman DS, Dietz WH, Tang R, Mensah GA, Bond MG, Urbina EM, Srinivasan S \& Berenson GS. The relation of obesity throughout life to carotid intima-media thickness in adulthood: the Bogalusa Heart Study. International Journal of Obesity and Related Metabolic Disorders 200428 159-166.

13 Wu DM, Chu NF, Shen MH \& Wang SC. Obesity, plasma high sensitivity c-reactive protein levels and insulin resistance status among school children in Taiwan. Clinical Biochemistry 200639 810-815.

14 Jarvisalo MJ, Harmoinen A, Hakanen M, Paakkunainen U, Viikari J, Hartiala J, Lehtimaki T, Simell O \& Raitakari OT. Elevated serum C-reactive protein levels and early arterial changes in healthy children. Arteriosclerosis, Thrombosis, and Vascular Biology 200222 1323-1328.

15 Steinberg D \& Witztum JL. Is the oxidative modification hypothesis relevant to human atherosclerosis? Do the antioxidant trials conducted to date refute the hypothesis? Circulation 2002105 2107-2111.

16 Garg A. Adipose tissue dysfunction in obesity and lipodystrophy. Clinical Cornerstone 20068 S7-S13.

17 Frayn KN. Adipose tissue and the insulin resistance syndrome. Proceedings of the Nutrition Society 200160 375-380.

18 Mlinar B, Marc J, Janez A \& Pfeifer M. Molecular mechanisms of insulin resistance and associated diseases. Clinica Chimica Acta 2007375 20-35.

19 Haque WA, Shimomura I, Matsuzawa Y \& Garg A. Serum adiponectin and leptin levels in patients with lipodystrophies. Journal of Clinical Endocrinology and Metabolism 2002872395.

20 Higashi Y, Sasaki S, Nakagawa K, Kimura M, Noma K, Sasaki S, Hara K, Matsuura H, Goto C, Oshima T, Chayama K \& Yoshizumi M. Low body mass index is a risk factor for impaired endothelium-dependent vasodilation in humans: role of nitric oxide and oxidative stress. Journal of the American College of Cardiology 200342 256-263. 
21 Krebs NF \& Jacobson MS. Prevention of pediatric overweight and obesity. Pediatrics 2003112 424-430.

22 Rolland-Cachera MF, Cole TJ, Sempe M, Tichet J, Rossignol C \& Charraud A. Body mass index variations: centiles from birth to 87 years. European Journal of Clinical Nutrition 1991 45 13-21.

23 Moreno LA, Rodríguez G, Guillén J, Rabanaque MJ, León JF \& Ariño A. Anthropometric measurements in both sides of the body in the assessment of nutritional status in prepubertal children European Journal of Clinical Nutrition 200256 1208-1215.

24 Foreman JR, Karlin JB, Edelstein C, Juhn DJ, Rubenstein AH \& Scanu AM. Fractionation of human serum lipoproteins by singlespin gradient ultracentrifugation: quantification of apolipoproteins B and A-1 and lipid components. Journal of Lipid Research 197718 759-767.

25 Belcher JD, Balla J, Balla G, Jacobs DR Jr, Gross M, Jacob HS \& Vercellotti GM. Vitamin E, LDL, and endothelium. Brief oral vitamin supplementation prevents oxidized LDL-mediated vascular injury in vitro. Arteriosclerosis and Thrombosis 199313 1779-1789.

26 Simon BC, Cunningham LD \& Cohen RA. Oxidized low density lipoproteins cause contraction and inhibit endothelium-dependent relaxation in the pig coronary artery. Journal of Clinical Investigation 199086 75-79.

27 Roberts LJ \& Morrow JD. Measurement of F(2)-isoprostanes as an index of oxidative stress in vivo. Free Radical Biology and Medicine 200028 505-513.

28 Lee BL, Chua SC, Ong HY \& Ong CN. High-performance liquid chromatographic method for routine determination of vitamins A and $\mathrm{E}$ and beta-carotene in plasma. Journal of Chromatography $1992 \mathbf{5 8 1} 41-47$.

29 Ford ES. C-reactive protein concentration and cardiovascular disease risk factors in children: findings from the National Health and Nutrition Examination Survey 1999-2000. Circulation 2003 108 1053-1058.

30 The fourth report on the diagnosis, evaluation, and treatment of high blood pressure in children and adolescents. Pediatrics 2004 $114555-576$.

31 Pignoli P, Tremoli E, Poli A, Oreste P \& Paoletti R. Intimal plus medial thickness of the arterial wall: a direct measurement with ultrasound imaging. Circulation 1986 74 1399-1406.

32 Raitakari OT, Juonala M, Kahonen M, Taittonen L, Laitinen T, Maki-Torkko N, Jarvisalo MJ, Uhari M, Jokinen E, Ronnemaa T, Akerblom HK \& Viikari JS. Cardiovascular risk factors in childhood and carotid artery intima-media thickness in adulthood: the Cardiovascular Risk in Young Finns Study. Journal of the American Medical Association $20032902277-2283$.

33 Davis PH, Dawson JD, Riley WA \& Lauer RM. Carotid intimalmedial thickness is related to cardiovascular risk factors measured from childhood through middle age: the Muscatine Study Circulation $20011042815-2819$.

34 Rosfors S, Hallerstam S, Jensen-Urstad K, Zetterling M \& Carlstrom C. Relationship between intima-media thickness in the common carotid artery and atherosclerosis in the carotid bifurcation. Stroke 199829 1378-1382.
35 Sorof JM, Alexandrov AV, Cardwell G \& Portman RJ. Carotid artery intimal-medial thickness and left ventricular hypertrophy in children with elevated blood pressure. Pediatrics 2003111 61-66.

36 Jarvisalo MJ, Jartti L, Nanto-Salonen K, Irjala K, Rönnemaa T, Hartiala JJ, Celermajer DS \& Raitakari OT. Increased aortic intimamedia thickness: a marker of preclinical atherosclerosis in high risk children. Circulation 2001104 2943-2947.

37 Tonstad S, Joakimsen O, Stensland-Bugge E, Leren TP, Ose L, Russell D \& Bonaa KH. Risk factors related to carotid intima-media thickness and plaque in children with familial hypercholesterolemia and control subjects. Arteriosclerosis, Thrombosis, and Vascular Biology 199616 984-991.

38 Hannon TS, Janosky J \& Arslanian SA. Longitudinal study of physiologic insulin resistance and metabolic changes of puberty. Pediatric Research $200660759-763$.

39 Abate N, Garg A, Peshock RM, Stray-Gundersen J, Adams-Huet B \& Grundy SM. Relationship of generalized and regional adiposity to insulin sensitivity in men with NIDDM. Diabetes 199645 1684-1693.

40 Abate N, Garg A, Peshock RM, Stray-Gundersen J \& Grundy SM. Relationships of generalized and regional adiposity to insulin sensitivity in men. Journal of Clinical Investigation $19959688-98$.

41 Garg A \& Misra A. Lipodystrophies: rare disorders causing metabolic syndrome. Endocrinology and Metabolism Clinics of North America 200433 305-331.

42 Yudkin JS. Abnormalities of coagulation and fibrinolysis in insulin resistance. Evidence for a common antecedent? Diabetes Care 1999 22 C25-C30.

43 Evans JL, Goldfine ID, Maddux BA \& Grodsky GM. Are oxidative stress-activated signaling pathways mediators of insulin resistance and beta-cell dysfunction? Diabetes 200352 1-8.

44 Morrow JD. Quantification of isoprostanes as indices of oxidant stress and the risk of atherosclerosis in humans. Arteriosclerosis, Thrombosis, and Vascular Biology 200525 279-286.

45 Pratico D, Iuliano L, Mauriello A, Spagnoli L, Lawson JA, Rokach J, Maclouf J, Violi F \& FitzGerald GA. Localization of distinct F2isoprostanes in human atherosclerotic lesions. Journal of Clinical Investigation $19971002028-2034$

46 Mohn A, Catino M, Capanna R, Giannini C, Marcovecchio M \& Chiarelli F. Increased oxidative stress in prepubertal severely obese children: effect of a dietary restriction-weight loss program. Journal of Clinical Endocrinology and Metabolism 200590 2653-2658.

47 Nigro J, Osman N, Dart AM \& Little PJ. Insulin resistance and atherosclerosis. Endocrine Reviews 200627 242-259.

Received 29 April 2009

Accepted 6 May 2009 